Multiples Myelom

\title{
Therapieergebnisse verbessern durch Proteasominhibition
}

\begin{abstract}
Beim multiplen Myelom steht eine Vielzahl von Therapieregimen und Substanzen zur Verfügung, um dem einzelnen Patienten eine möglichst effektive Behandlung zu bieten. Dem Proteasominhibitor Bortezomib kommt hier ein hoher Stellenwert zu.
\end{abstract}

In der täglichen Praxis spielen für die Therapieentscheidung vor allem der Remissionsdruck und eventuelle Begleiterkrankungen eine Rolle, aber auch die Zytogenetik, Vortherapien sowie Alter und Wünsche des Patienten, so Dr. Hans Salwender, Hamburg. Die Kombination Bortezomib (Velcade ${ }^{\circledast}$ ) plus Melphalan/Prednison (VMP) hat sich in der VISTA-Studie als sehr effizient für die Behandlung älterer, nicht für eine Hochdosistherapie geeigneter Patienten erwiesen. Nach 3-jährigem Follow-up lebten im VMP-Arm noch $68 \%$ der Patienten versus 54\% im MP-Arm, das mediane Gesamtüberleben war unter VMP noch nicht erreicht versus 43,1 Monate unter MP ( $p=0,0008$; Mateos MV et al., 2010, J Clin Oncol 28:2259-2266). Hervorzuheben ist die hohe Rate kompletter Remissionen in der VMP-Gruppe (30\% versus 4\%; San Miguel JF et al., 2008, N Engl J Med 359: 906-917), da auch bei älteren Patienten das Ansprechen

positiv mit der Prognose korreliert (Gay F et al., 2011, Blood 117: 3025-3031).

Aber auch in anderen Therapiesituationen hat sich der Einsatz von Bortezomib als vorteilhaft erwiesen, wie z.B. im Rahmen von Hochdosisschemata in der Induktions- und Erhaltungstherapie (Abb.)

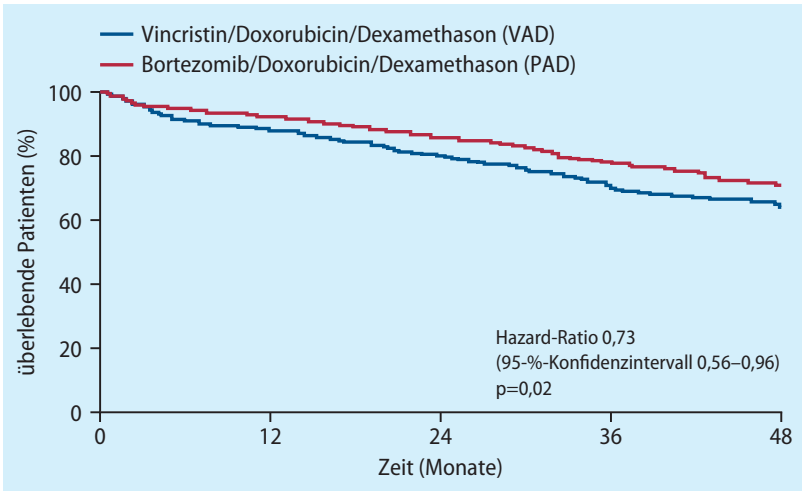

nach Sonneveld P et al., 2010, ASH: \#40

HOVON-65/GMMG-HD4-Studie: Gesamtüberlebensvorteil mit Bortezomib-haltiger Induktions- und Erhaltungstherapie

\section{Nebenwirkungsmanagement}

Bortezomib hat eine etwas höhere Neurotoxizität als die meisten anderen beim Myelom verwendeten Substanzen. Dies stellte eine Herausforderung dar, da schon Grad-2Neuropathien mit Schmerzen eine Dosisreduktion erfordern, wie PD Dr. Martin Kropff, Münster, erläuterte. Inzwischen sind zwei gut praktikable Wege bekannt, die peripheren Neuropathien deutlich zu vermindern bei gleich bleibender Effektivität. Dies kann durch Reduktion der Bortezomib-Dosis im VMP-Schema von zweimaliger auf einmal wöchentliche Gabe geschehen (Palumbo A et al., 2010, ASH:\#620), aber auch die subkutane anstelle der bisher üblichen intravenösen Applikation führt zu einer besseren Verträglichkeit ohne Wirkungsverlust (Moreau P et al., 2011, Lancet Oncol 12: 431-440).

Eine weitere bekannte Nebenwirkung ist dieHerpes-zoster-Reaktivierung. Sielässt sich durch eine antivirale Prophylaxe (z.B. Aciclovir) zuverlässig verhindern, so Kropff. $C L$

Quelle: Jahrestagung der Deutschen, Österreichischen und Schweizerischen Gesellschaften für Hämatologie und Onkologie 2011, Basel/Schweiz, Satellitensymposium; Janssen-Cilag GmbH

\section{Chronische lymphatische Leukämie}

\section{Therapie mit Bendamustin für die Mehrzahl der Patienten geeignet}

Für die Wirksamkeit und Verträglichkeit von Bendamustin in der Behandlung der chronischen lymphatischen Leukämie (CLL) gibt es zahlreiche gute Studiendaten. Das schlägt sich auch im Behandlungsalltag nieder.

Basierend auf den Ergebnissen der CLL8Studie gilt Fludarabin/Cyclophosphamid (FC) plus Rituximab (FC-R) als Standard für die Behandlung der CLL (Hallek $M$ et al., 2010, Lancet 376: 1164-1174). Aufgrund eines erhöhten Risikos für hämatologische Toxizitäten wird der Einsatz von FC-R laut Prof. Dr. Wolfgang Knauf, Frankfurt/Main, im derzeit gültigen Therapiealgorithmus der Deutschen CLL-Studiengruppe (DCLLSG) aber nur auf fitte Patienten beschränkt. Erste Ergebnisse der CLL10-Studie zeigen nun, dass die Kombination Bendamustin plus Rituximab (B-R) bei verminderter Toxizität eine vergleichbar effektive Alternative zu FC-R ist (Fischer Ket al., 2009, Blood 114: \#114).

Die meisten Patienten mit CLL weisen einen eingeschränkten Gesundheitsstatus auf. Für diese Patienten werden Therapieoptionen mit hoher Wirksamkeit und gleichzeitig geringer bzw. kalkulierbarer Toxizität gesucht. Beides konnten Knauf et al. für die Behandlung mit Bendamustin zeigen: Im Vergleich zu Chlorambucil ergaben sich u.a. eine signifikant höhere Gesamtremissionsrate $(68 \%$ versus $31 \% ; p<0,0001)$ und ein signifikant längeres progressionsfreies Überleben (median 21,6 Monate versus 8,3 Monate; $\mathrm{p}<0,0001$; Knauf WU et al., 2009, J Clin Oncol 27: 4378-4384).

\section{Bendamustin-basierte Therapie im Behandlungsalltag etabliert}

Aktuelle Daten des deutschen Tumorregisters Lymphatische Neoplasien zeigen, dass B-R sowohl in der First- als auch in der Second-line-Therapie das meist eingesetzte Schema zur Behandlung der CLL in der hämato-onkologischen Praxis ist.

In den aktuellen Leitlinien der Deutschen Gesellschaft für Hämatologie und Onkologie wird die Kombination B-R bei aktiver Hämolyse und für Patienten mit Niereninsuffizienz als Alternative zu FC-R empfohlen (www. dgho.de/onkopedia).

Quelle: Jahrestagung der Deutschen, Österreichischen und Schweizerischen Gesellschaften für Hämatologie und Onkologie 2011, Basel/Schweiz, Satellitensymposium und Informationen der Mundipharma GmbH 